\title{
Space Debris Removal with an Ion Beam Shepherd Satellite: target-plasma interaction
}

\author{
M. Merino*, E. Ahedo ${ }^{\dagger}$, C. Bombardelli ${ }^{\ddagger}$, H. Urrutxua ${ }^{\S}$ and J. Peláez ${ }^{\dagger}$ \\ Universidad Politécnica de Madrid, 28040 Madrid, Spain \\ L. Summerer \\ ESA Advanced Concepts Team, The Netherlands
}

\begin{abstract}
A novel concept for active space debris removal known as Ion Beam Shepherd (IBS) which has been recently presented by our group is investigated. The concept makes use of a highly collimated ion beam to exert the necessary force on a generic debris to modify its orbit and/or attitude from a safe distance in a controlled manner, without the need of docking. After describing the main characteristics of the IBS system, some of the key aspects of thruster plasma and its interaction with the debris are studied, namely, (1) the modeling of the expansion of an plasma beam, based on the quasi-selfsimilarity exhibited by hypersonic plumes, (2) the characterization of the force and torque exerted upon the target debris, and (3) a preliminary evaluation of other plasma-body interactions.
\end{abstract}

\section{Introduction}

Space debris are becoming a serious threat for the continued exploitation of space at Low Earth and Geostationary Orbits (LEO and GEO) altitudes. Debris impacts occur at an average relative velocity of $10 \mathrm{~km} / \mathrm{s}$, meaning that a regular $1 \mathrm{~cm}$ object can pierce any vehicle but the most highly shielded. Current studies $^{1}$ predict that the number of debris in the orbits of interest will continue to increase unavoidably during the next years even in the absence of future launches as the consequence of collisions and explosions. Although catastrophic collision probabilities are currently low, there is fear for a plausible chain-reaction of collisions between debris, which could result in hundreds of thousands of pieces that would render such orbits unusable in practice for decades or centuries (scenario known as Kessler Syndrome ${ }^{2}$ ). These catastrophic possibilities underline the need for Active Debris Removal (ADR) actions, i.e., displacing space debris from crowded orbits using a dedicated orbiting and/or ground based facility.

ADR is, however, an expensive and risky task. Typically, a target space debris has to be docked by another spacecraft before a deorbiting maneuver takes place. Docking with a non-cooperative target, which, in addition, may exhibit a chaotic attitude motion, poses a great technology problem. Recently, our research team has proposed a novel contactless debris removal strategy, ${ }^{3,4}$ which can greatly reduce the complexity, costs and risks of an active removal campaign. The idea is based on an Ion Beam Shepherd (IBS) spacecraft, which employs a high-specific-impulse ion beam to transmit sufficient momentum to the debris to deorbit it, avoiding the docking problem. Concurrently, similar concepts have been independently proposed by JAXA ${ }^{5}$ and CNES. ${ }^{6}$

The IBS concept consists of three critical aspects, namely: (1) the generation and evolution of an adequate ion beam, (2) the plasma-body interactions that take place at the surface of the target debris and in the neighboring plasma due to the presence of the immersed body, and (3) the relative orbital dynamics of the system. This paper contains a preliminary analysis of the ion beam physics and the different aspects of the ion beam-body interactions of the proposed ADR system. This is the first of a set of two companion articles

\footnotetext{
*Student, AIAA Student Member (mario.merino@upm.es).

$\dagger$ Professor, AIAA Member.

${ }^{\ddagger} \mathrm{PhD}$.

§Student, AIAA Student Member.
} 


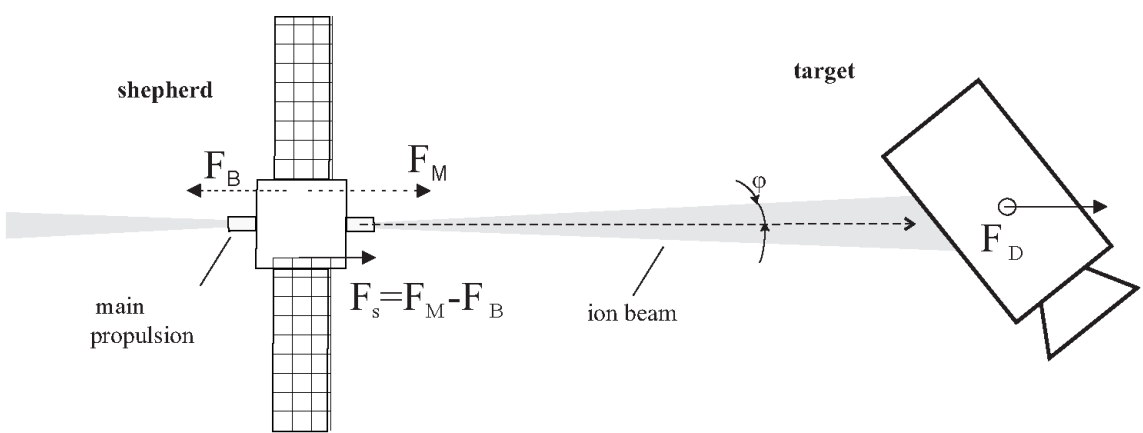

Figure 1. Schematic of ion beam shepherd satellite deorbiting a space debris

describing the IBS concept, the second one (Ref. 7) covering the relative orbit dynamics of the system, the attitude dynamics of the debris under the action of the ion beam, and the control strategies of the IBS.

After describing the IBS concept in section II, the main requirements for the ion beam are discussed in section III. Identifying the key parameters that govern the divergence of the ion beam is paramount for this application, since low divergence increases the fraction of ion flux reaching the body, and/or permits to act on the debris from a larger distance. Then, in section IV, different types of beam models are examined, and after comparing their qualities, a quasi-selfsimilar plasma plume model is used to characterize the plasma expansion. Section V covers the calculation of the delivered force and torque on the body, based on the ion beam model and a few simplifying, conservative assumptions. In section VI, a first evaluation of secondary plasma-body interactions is performed, discussing (1) relative charging of the IBS satellite and the debris, (2) plasma plume modification by the immersed body, (3) backscattering and sputtering from the material surface, and (4) backflow contamination of the shepherd satellite. Finally, the most salient conclusions are gathered in section VII.

\section{The Ion Beam Shepherd}

The proposed ADR idea consists in bringing into orbit the IBS spacecraft, which will chase and rendezvous with a preselected set of target debris. Once positioned adjacent to its target - and while maintaining a close formation flying, - a low-divergence, high-specific-impulse ion beam will be directed towards the body to exert a decelerating force on it, in order to deorbit the debris (or rise it up to a disposal orbit), hence avoiding the technological challenge of physically docking to an uncooperative target. The shepherd spacecraft is equipped with a main propulsion system to counteract the reaction force of the beam-generator thruster, allowing to keep a constant distance between the space debris constant throughout the deorbit (or reorbit) process. The IBS concept is illustrated in figure 1. An interesting capability of the concept is the possibility to perform multi-target deorbiting campaigns with a single IBS. After concluding with the first deorbiting maneuver, the IBS would then rendezvous with the next target, and continue its removal mission.

The ion beam acts basically as a physical link between the two bodies, and when properly controlled, it works as a stable, spring-like mechanism to convey the required forces to the debris. The two-body system as a whole is then propelled by the main propulsion system. Using this ion physical link to act upon the target entails an additional propellant consumption, needed to produce the mediating ion beam. This is the necessary price to avoid the need of docking with the debris. The employment of high-specific impulse thrusters allows to keep propellant usage low.

In order to maintain a constant distance, the thrust produced by the main propulsion system, $F_{M}$, must be related to the force of the beam-generator thruster, $F_{B}$, through

$$
F_{M}=F_{B}\left(1+\eta_{B} \frac{m_{I B S}}{m_{D}}\right)
$$

where $m_{I B S}, m_{D}$ are the mass of the IBS satellite and the debris, respectively, and $\eta_{B}$ is the momentum transfer efficiency achieved with the primary beam, defined as the ratio between the force exerted on the 


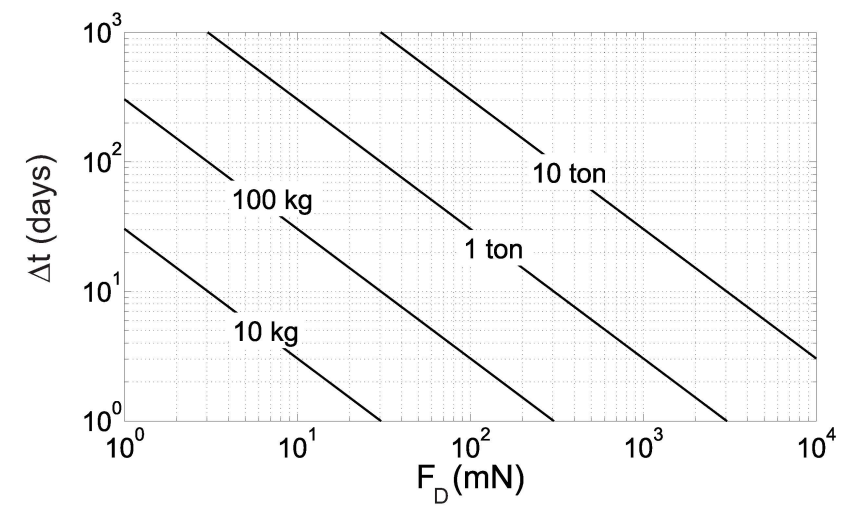

Figure 2. Deorbiting time for different values of the space debris mass and the (tangential) force $F_{D}$ exerted on the debris, for a low-thrust transfer from $1000 \mathrm{~km}$ to $500 \mathrm{~km}$ altitude circular orbit.

debris $F_{D}$ and the thrust $F_{B}$ :

$$
\eta_{B}=\frac{F_{D}}{F_{B}}
$$

Momentum losses are due to part of the plasma beam not being intercepted by the target debris. In order to obtain an efficiency $\eta_{B} \simeq 1$, the separation between shepherd and debris must be low enough, and low-divergence beams need to be employed.

Most of LEO debris occupy the altitudes between 800 and $1000 \mathrm{~km}$, have near-circular orbits, and large inclinations $(\simeq 80 \mathrm{deg})$. A preliminary performance study conducted in Ref. 3 has shown that a typical 2-ton LEO debris can be deorbited in a 3-4 months with an ion beam with $F_{B} \simeq 100 \mathrm{mN}$, on board of a shepherd satellite of a few hundred kilograms. Figure 2 displays information on the deorbiting time for different debris masses and forces.

\section{Propulsion requirements}

The whole IBS concept relies on the ability to transmit a sufficient deorbiting force to a distant body with an ion beam. It is therefore critical to analyze the main requeriments for the propulsion system, and discuss the different alternatives that can be used to generate the beam. As highlighted in the previous section, to achieve this in a highly efficient manner, we require a plasma source able to generate (1) a high specific impulse, $I_{s p}$, while having a high thrust efficiency, $\eta_{T}=I_{s p} F_{B} /\left(2 P_{a B}\right), P_{a B}$ being the available power for the ion beam source, and (2) a low divergence plasma jet.

The specific impulse (or effective beam velocity) of the source is a measure of propellant utilization, a larger value meaning lower consumption for a same mission $\Delta V$. The available power onboard, $P_{a}$, and the deorbiting design time, $\Delta t$, set nevertheless a constrain to the maximum $I_{s p}$. Assuming constant $F_{B}$ and $\eta_{B}$, and that both the main propulsion system and the ion beam source have the same $I_{s p}$ and thrust efficiency $\eta_{T}$, this constrain renders

$$
I_{s p}=\frac{2 \eta_{T} \eta_{B} P_{a} \Delta t}{\Delta V\left(m_{D}+\eta_{B} m_{I B S}\right)} .
$$

This expression manifests the importance of the total embarked power $P_{a}$ and a large thrust efficiency $\eta_{T}$ for a swift and efficient deorbiting campaign.

The ability to control thrust produced by the device is also a desired feature to allow controlling the distance between the two orbiting bodies.

The other main factor paramount for this application is the divergence angle of the beam. Given a working distance from the shepherd satellite to a certain target debris, the divergence angle defines the amount of plasma intercepted by the object, thereby determining the beam momentum transfer efficiency $\eta_{B}$. The smaller the divergence angle, the higher $\eta_{B}$ for a given separation distance, or, equivalently, the longer the IBS separation distance can be for a fixed value of $\eta_{B}$.

Finally, the characteristics of existing plasma thrusters and the their technological maturity have also to be taken into account. The two most trusted and successful types of advanced electric propulsion are 
ion engines and Hall effect thrusters, which are nowadays fully-developed, space-tested, and commercially available in the power range $500-5000 \mathrm{~W}$.

The practical differences between both types of thruster are: ${ }^{8}$

- The larger divergence angle of the plasma plume of Hall thrusters ( $40 \mathrm{deg})$ compared with that of ion engines (typically $<20 \mathrm{deg}$ ).

- The higher overall efficiency of ion engines $(\sim 65-80 \%)$, compared with Hall thrusters $(\sim 50 \%)$.

- The larger specific impulse of ion engines ( $3000 \mathrm{~s})$, with respect to Hall thrusters (1500-2500 s, normally).

- Hall thrusters are more compact and lighter for the same thrust than ion engines.

- Ion engines rely on more complex power processing units, and the thrust they produce is limited by space charge effects between grids, where electrons are absent.

Current thrusters of both types with $2-3 \mathrm{~kW}$ provide around $100 \mathrm{mN}$ thrust. All these differences, remarkably the smaller divergence angle, suggest that ion engines are more appropriate for the IBS. Nevertheless, both types of engines, as well as other well-established devices such as arcjets, can be potentially employed in the IBS. Idem can be said of new and under-development electric propulsion technologies, such as helicon thrusters with tunable magnetic nozzles ${ }^{9}$ or magnetoplasmadynamic thrusters ${ }^{10}{ }^{10}$ whose performances and characteristics might prove to be competitive for this application.

\section{Plasma beam characterization}

The hypersonic beam used to exert the deorbiting force on the debris is a central part of the IBS concept. An accurate model of the plasma expansion is required to (1) understand the plume physics, (2) identify the key parameters that control the evolution of the beam and its divergence angle, and (3) calculate the IBS performances (force, torque and efficiencies).

The plume of ion and Hall effect thrusters can be described as composed of two different regions. Firstly, there is the near-field region, reaching a few thruster radii out of the engine exit, in which plasma inhomogeneities and thruster electromagnetic fields dictate the initial formation of the plasma plume. Secondly, there is the far-field plume, where the plasma inhomogeneities have died out, the plasma profile is singlepeaked (in Hall thrusters, the density profile is already single-peaked after only 2 radii $^{11}$ ), and influence from the thruster electromagnetic fields is negligible. In this region, plume evolution is predominantly governed by the residual internal pressure of the plasma.

Modeling of the near-field is highly complex, due to all the intertwined processes taking place. The phenomena in this region determine the initial divergence angle of the ion beam in the far-field, and in the case of Hall effect thrusters in this zone also the final stages of ion acceleration take place. For the IBS application, where the target object is going to be located well into the far-field region, our interest in the near-field is limited to the establishment of the initial divergence angle, and attention will be focused on the far-field.

There are a number of different models for the far field plasma plume, each of them having different levels of complexity and accuracy. As an overview, they can be classified in increasing level of complexity as:

1. Point-source approximations: the plume is modeled analytically as a conical expansion from a point source, neglecting thermal expansion downstream. ${ }^{12,13}$ Models which fall in this category provide a coarse approximation of the plasma properties.

2. Quasi-selfsimilar expansion models: The hypersonic flow is assumed self-similar, and plasma profiles are derived from a set of initial hypotheses. In this way semi-analytical models are obtained. They capture the expansion due to thermal effects and the influence of the relevant thruster parameters. ${ }^{14-16} \mathrm{We}$ have found out that the self-similar assumption is only an approximation, since the full fluid equations do not admit, in general, such a solution without the addition of a simplifying hypothesis, but the error incurred by it is small for highly supersonic flows, i.e., for initial Mach numbers verifying $M_{0} \gg 1$.

3. Full numerical models: This group encompasses everything from the integration of simplified fluid equations to Particle-in-Cell simulations and other advanced models. ${ }^{17-19}$ The complexity is higher, 
but they take into account the influence of secondary effects like ambient plasma effects, chargeexchange (CEX) ions, and background pressure for tests in vacuum chambers. ${ }^{15,20}$ A current field of application of these models is the study of details of the plasma-surface interactions with solar panels and other spacecraft parts.

Arguably, the models belonging to the first group are comparable in computational cost with those of the second group, although the latter provide much better accuracy. The third group, however, necessarily requires much larger computational resources. Hence, for this preliminary stage of analysis of the IBS concept, quasi-selfsimilar models (QSSM) have been chosen as the best compromise solution between complexity and difficulty, meaning that secondary effects will be neglected in the simulations.

In the following, a slightly modified version of the Ashkenazy and Fruchtman model ${ }^{16}$ is briefly described and used. A detailed discussion and generalization of different hypersonic plasma plume QSSM, including the study of the influence of the environment in the development of the plume, will be soon released (Ref. 21).

A steady-state, non-rotating plasma, formed by single-charged cold ions and hot electrons $\left(p_{i} \ll p_{e}\right.$, with $p_{i}$ and $p_{e}$ the ion and electron pressure, respectively), in the absence of collisional or magnetic effects will be modeled. Local current ambipolarity, $\boldsymbol{j}=0$, will be assumed in the axysimmetric plume. The fluid equations of such a plasma comprise the ion $(i)$ and electron $(e)$ continuity and momentum equations. Expressed in cylindrical coordinates $(z, r, \theta)$, with $z$ along the plume axis and the origin at an arbitrary initial plane inside the far field plume, ion equations read:

$$
\begin{gathered}
u_{z i} \frac{\partial \ln n}{\partial z}+u_{r i} \frac{\partial \ln n}{\partial r}+\frac{\partial u_{z i}}{\partial z}+\frac{1}{r} \frac{\partial\left(r u_{r i}\right)}{\partial r}=0, \\
u_{z i} \frac{\partial u_{z i}}{\partial z}+u_{r i} \frac{\partial u_{z i}}{\partial r}+\frac{e}{m_{i}} \frac{\partial \phi}{\partial z}=0, \\
u_{z i} \frac{\partial u_{r i}}{\partial z}+u_{r i} \frac{\partial u_{r i}}{\partial r}+\frac{e}{m_{i}} \frac{\partial \phi}{\partial r}=0,
\end{gathered}
$$

where $u_{z i}, u_{r i}$ are the ion velocity components in the $z, r$ direction. The electron $(e)$ momentum equation is:

$$
\frac{1}{n} \nabla p_{e}-e \nabla \phi=0
$$

where electron inertia has been neglected. Finally, an equation of state, such as $p_{e}=n T_{e}$ for an ideal gas (electron temperature expressed in energy units), and a barotropy relation, i.e., one allowing to express $p_{e}$ as a function of $n$, are also needed:

$$
p_{e}=p_{e}(n) .
$$

For our purposes, it suffices to consider that the electrons evolve isothermally along the plume, $T_{e}=$ const. Experimental measurements of the electron temperature reveal that variations in the far field plume are small. ${ }^{22}$

These equations can be normalized using $T_{e}, m_{i}$ (ion mass), $e$ (elementary electric charge), $n_{0}$ (particle density at the chosen origin), and the characteristic length $R_{0}$ that can be defined as the radius of the plasma tube at $z=0$ containing a specified fraction of the total ion flux of the plume (e.g., the tube containing $95 \%$ of the flow, since this tube is used conventionally to define the plume divergence angle). In the following, dimensionless variables will be denoted with a tilde, e.g., $\tilde{\phi}=e \phi / T_{e}$ or $\tilde{u}_{z i}=u_{z i} / \sqrt{T_{e} / m_{i}}$, where $c_{s}=\sqrt{T_{e} / m_{i}}$ is the ion sound velocity.

QSSM are based in the assumption that all ion streamlines, $\left(z_{i}, r_{i}\right)$, expand likewise, i.e., their expansion can be expressed through a dimensionless self-similarity function $h(\tilde{z})$,

$$
\tilde{r}_{i}\left(\tilde{z}_{i}\right)=\tilde{r}_{i 0} h\left(\tilde{z}_{i}\right),
$$

where $r_{i 0}$ is their initial radial position in the chosen reference plane $\tilde{z}=0$, where $h(0)=1$. It will be useful in the following to define a new coordinate system, $\zeta, \eta$, with

$$
\zeta=\tilde{z}, \quad \eta=\frac{\tilde{r}}{h} .
$$

The set of equations 4-8 is in general incompatible with the self-similarity assumption, Eq. 9. To proceed, it is necessary to substitute one of these equations with an adequate assumption. When the flow is 
highly hypersonic in the axial direction, with the axial Mach number $M_{z}=\tilde{u}_{z i} \gg 1$, Eq. 5 shows that axial velocity variations along streamlines are small compared to velocity itself: $\Delta M_{z} / M_{0} \sim \Delta \ln n / M_{0}^{2} \ll 1$, where $M_{0}=u_{0} / \sqrt{T_{e} / m_{i}}$ is the Mach number at the origin. Equation 5 is therefore substituted by $\tilde{u}_{z i}=$ const along ion streamlines ( $\eta=$ const). Assuming an initial conical velocity profile at the $\zeta=0$ plane, the expressions for the velocity components, plasma density and $h$ function become:

$$
\begin{gathered}
\tilde{u}_{z i}=\frac{M_{0}}{\sqrt{1+\eta^{2} / \tilde{d}^{2}}}, \quad \tilde{u}_{r i}=\eta h^{\prime} \tilde{u}_{z i}, \\
\tilde{n}=\frac{1}{h^{2}\left(1+\eta^{2} / \tilde{d}^{2}\right)^{\tilde{d}^{2} / \sigma^{2}+1 / 2}}, \\
h^{\prime}=\sqrt{C^{2} \ln h+\tilde{d}^{-2}}, \quad \text { i.e. } \quad \int_{1}^{h} \frac{d h_{1}}{\sqrt{C^{2} \ln h_{1}+\tilde{d}^{-2}}}=\zeta,
\end{gathered}
$$

with $\tilde{d}$ the dimensionless distance of this plane to the cone vertex used to define the flow (notice that $\tilde{d}^{-1}$ coincides with the flow tangent at $\zeta=0, \eta=1)$, and $C^{2}=\left(4+2 \sigma^{2} \tilde{d}^{-2}\right) /\left(\sigma^{2} M_{0}^{2}\right)$. The parameter $\sigma$ allows to adjust the flow so that $95 \%$ of the total ion flux is contained in the $\eta \leq 1$ streamtube (i.e., the tube inside $r=R_{0}$ at $z=0$ ). Note that in Ref. 16 a different definition of $R_{0}$ is used that does not coincide with the initial radius of the $95 \%$-flux streamtube. The value of $\sigma$ can be obtained integrating the flow and imposing the $95 \%$-flux condition,

$$
1-\left(1+\frac{1}{\tilde{d}^{2}}\right)^{-\tilde{d}^{2} / \sigma^{2}}=0.95
$$

The model therefore depends on the centerline Mach number $M_{0}$ and the initial divergence parameter $\tilde{d}$. The value of these two parameters defines the divergence of the plasma beam, as shown in figure 3, where a reference streamline has been plotted for different values of these two parameters. These two parameters govern the self-similarity function $h$ (see Eq. 13). The large influence of the Mach number stems from the role of the residual electron pressure, which tends to continue opening the beam downstream, making the plume non-conical: the larger the Mach number, the smaller thermal energy (and pressure) becomes when compared to kinetic energy, since

$$
M_{0}^{2}=\frac{m_{i} u_{0}^{2}}{T_{e}},
$$

and hence the less the divergence increase rate is. This suggests that the IBS concept would benefit from highly hypersonic beams. This can be further expressed as ion beams having a large axial velocity $u_{0}$ and heavier ions $\left(m_{i}\right)$, plus a low electron temperature $T_{e}$. Naturally, the lower the initial divergence angle, the lower the divergence is globally. Initial divergences as low as $10 \mathrm{deg}$ or smaller are attainable with SoA ion thrusters ${ }^{23,24}$ and it is expected that this value could be further decreased if this criterion became the central one in the design of thruster ion optics. ${ }^{5}$

The functions $\tilde{u}_{z i}, \tilde{u}_{r i}$ and $\tilde{n}$ are displayed in figure 4 , together with the $h$ function that defines ion streamlines. The relative local error $\varepsilon$ committed by not fulfilling Eq. 5 can be defined as:

$$
\varepsilon=\frac{\tilde{u}_{z i} \partial \tilde{u}_{z i} / \partial \tilde{z}+\tilde{u}_{r i} \partial \tilde{u}_{z i} / \partial \tilde{r}+\partial \ln n / \partial \tilde{z}}{\tilde{u}_{z i}^{2}}=\frac{2}{M_{0}^{2}} \frac{h^{\prime}}{h}\left[\left(\frac{1}{\sigma^{2}}-\frac{\tilde{d}^{-2}}{2}\right) \eta^{2}-1\right],
$$

where it is clear that the error is $\varepsilon \propto 1 / M_{0}^{2}$ so that it decreases fast as $M_{0}$ increases (i.e., for hypersonic flows). $\varepsilon$ also decreases downstream, as $h^{\prime} / h$ is a monotonic decreasing function. Except when $\tilde{d}=\sigma / \sqrt{2}$, there is one streamline

$$
\eta=\left(1 / \sigma^{2}-\tilde{d}^{-2} / 2\right)^{-1 / 2}
$$

for which the axial momentum equation is identically satisfied and the local error $\varepsilon$ cancels out; for any other value of $\eta$, error is not null. The error increases indefinitely for $\eta \rightarrow \infty$ except again for $\tilde{d}=\sigma / \sqrt{2}$. Nevertheless, the large error committed for large $\eta$ is irrelevant for the application at hand, since we are interested in the core of the plume, $\eta \lesssim 2$, where the practical totality of momentum flux is concentrated. The plasma at large angles from the centerline is so rarefied that its momentum can be conservatively neglected. The error has been plotted in figure 5 for two values of $\tilde{d}$. 
(a)

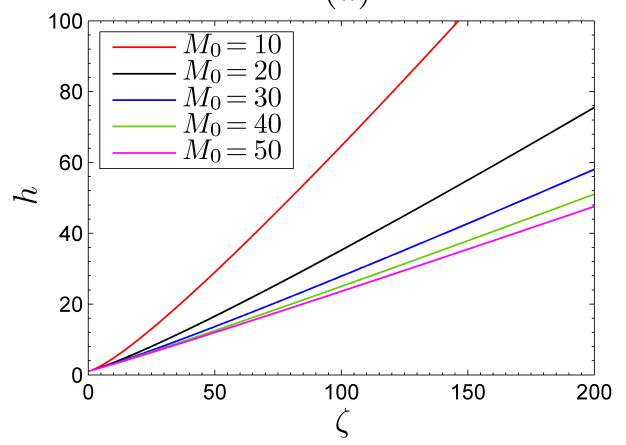

$(b)$

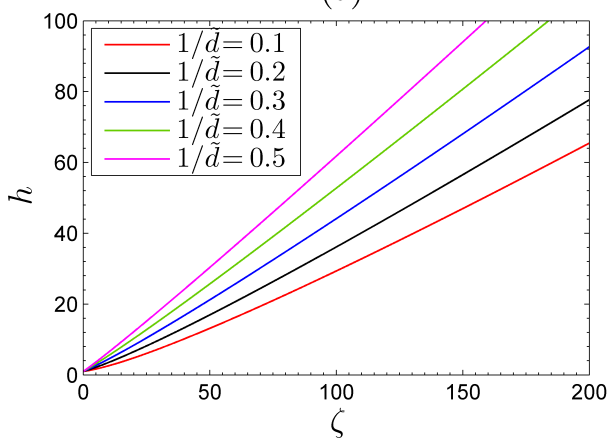

Figure 3. Influence of the Mach number $M_{0}$ (figure a) and the initial divergence tangent $\tilde{d}$ (figure b) on the $h$ function, which defines the ion streamlines of the flow. In figure $\mathbf{a}, \tilde{d}^{-1}=0.2$, and in figure $\mathbf{b}, M_{0}=20$.
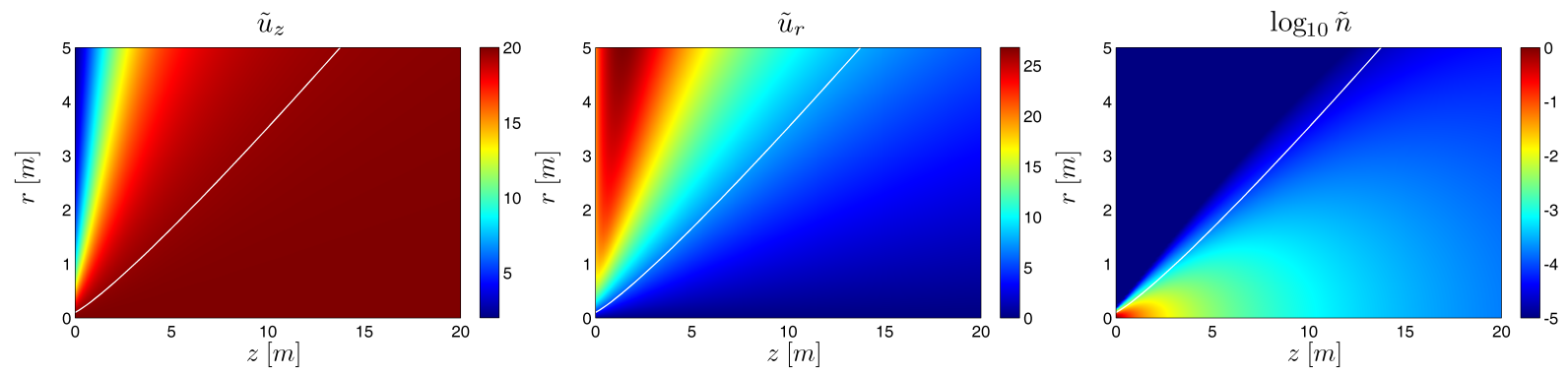

Figure 4. Dimensionless axial and radial velocities, $\tilde{u}_{z i}$ and $\tilde{u}_{r i}$, and the decimal logarithm of the plasma density $\log _{10} \tilde{n}$. These plots represent the $R_{0}=0.1 \mathbf{m}, \delta_{0}=0.2$, and $M_{0}=20$ (representative of $u_{z 0}=38000$ $\mathrm{m} / \mathrm{s}$ and $T_{e}=5 \mathrm{eV}$ for Xenon) case. The value of $\sigma$ has been chosen so that $\eta=1$ (in white) represents the streamtube of $95 \%$ flux. Notice that such line is not straight due to the residual pressure continuing to expand the plasma.

\section{Momentum transmission to the target body}

The model described in section IV can be used to evaluate the momentum delivered to a target debris by the plasma beam. This momentum has two basic contributions, due to (1) high energy ions impacting directly upon the debris, and (2) the electron pressure, which for an hypersonic beam is negligible (see Eq. 15). As ions reach the surface of the debris, they briefly penetrate the material lattice for a few nanometers, neutralize, and then after an accommodation time are rejected by the material binding forces back into space. Debris material sputtering can occur. The contribution to transferred momentum of the rejected propellant atoms and the sputtered materials is negligible, as will be shown in section VI. Hence, the delivered force $\boldsymbol{F}$ and torque $\boldsymbol{N}$ can be conveniently modeled assuming a perfectly inelastic collision of the incoming ions against the surface of the debris,

$$
\begin{gathered}
\boldsymbol{F}=\int_{S_{b}} m_{i} n \boldsymbol{u}_{i}\left(-\boldsymbol{\nu} \cdot \boldsymbol{u}_{i}\right) d S, \\
\boldsymbol{N}=\int_{S_{b}}\left(\boldsymbol{r}-\boldsymbol{r}_{G}\right) \times m_{i} n \boldsymbol{u}_{i}\left(-\boldsymbol{\nu} \cdot \boldsymbol{u}_{i}\right) d S,
\end{gathered}
$$

where $S_{b}$ is the surface of the debris illuminated by the ion beam, $\boldsymbol{\nu}$ is the outward-pointing unit vector perpendicular to the area element, $\boldsymbol{u}_{i}$ is the velocity vector of the incoming ions, $n$ their local density, and $\boldsymbol{r}-\boldsymbol{r}_{G}$ the position vector with respect to the center of mass of the debris. Figure 6 presents a sketch of the axial and a radial forces and the torque created by the ion beam.

These actions and the momentum transfer efficiency depend strongly on the size of the debris, its position within the plume, and in general also its attitude. Keeping a sufficiently small distance to the debris is paramount to maintain a momentum transfer efficiency near unity, $\eta_{B} \simeq 1$, so close formation flying (within 
(a)

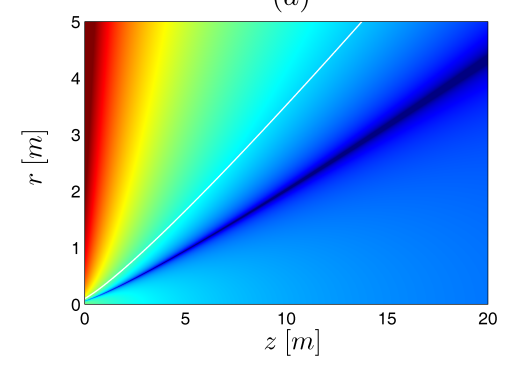

(b)

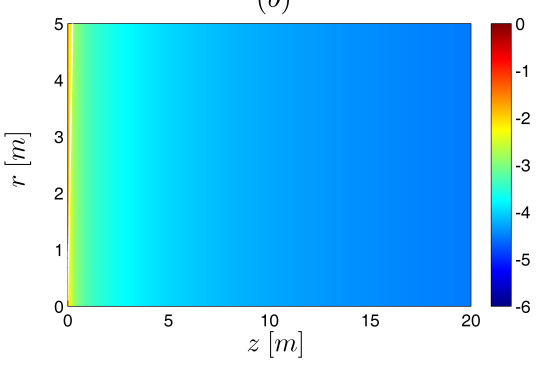

Figure 5. Logarithm of the relative error $\log _{10}|\varepsilon|$ as defined in equation 16. The represented simulations use $R_{0}=0.1 \mathrm{~m}, u_{0}=38000 \mathrm{~m} / \mathrm{s}$ at the origin and $T_{e}=5 \mathbf{e V}$, which for Xenon mean a Mach number at the origin $M_{0}=20$. Figure (a) is calculated for an initial divergence tangent $\tilde{d}^{-1}=0.2$, while (b) employs the unrealistic case $\tilde{d}^{-1}=\sqrt{2} / \sigma=19.97$ to show the case with $\eta$-independent error. The value of $\sigma$ has been chosen so that $\eta=1$ (in white) represents the streamtube of $95 \%$ flux, showing that case (b) does not represent a feasible/useful scenario for the IBS application.



Figure 6. Schematic showing the axial force $F_{z}$, radial force $F_{r}$ and torque $N$ transmitted to a spherical debris by the IBS beam.

10-15 $\mathrm{m}$ of the object for medium-sized debris) is a requirement of the IBS concept. Characterization of momentum transfer efficiency for a spherical debris is illustrated in figure 7.

The calculation of the forces and torques defined in Eqs. 17 and 18 is performed numerically with an inhouse developed general purpose simulation tool named IBIS (from Ion Beam Interaction Simulator). This program, which integrates the beam profile from the QSSM and the attitude and relative orbital dynamics of the two-body system, has proven to be a useful analysis tool for the study of the IBS concept and its performances.

The IBIS software employs hybrid-code, and is capable of simulating the interaction of a plasma plume with a body of arbitrary shape, provided its geometry. The computation of the forces and torques is made based on FEM algorithms, while a built-in algorithm deals with shadowing effects. As mentioned, a study of the relative orbital dynamics and debris attitude evolution in a model deorbiting mission with the IBIS software will be soon presented (Ref. 7).

\section{Other plasma-body interactions}

Besides transferring the required momentum to the target body, the plasma beam gives rise to a number of phenomena in its interaction with the debris, whose importance needs to be assessed. In this preliminary analysis we are chiefly concerned with (1) the electrostatic charging and interplay of the shepherd-plasmadebris system, (2) the modification of the plasma plume by the object, (3) the behavior and effects of ions when impinging on the target surface, and (4) the contamination of the shepherd satellite due to backflowing xenon and sputtered debris material. This section provides qualitative evaluation of all these aspects of the plasma-debris interaction. 


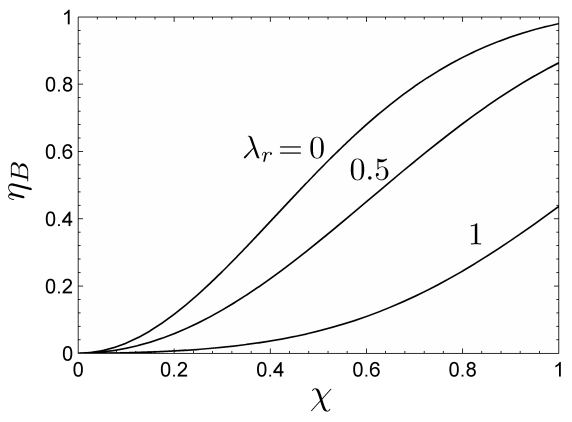

Figure 7. Momentum transfer efficiency $\eta_{B}$ for a spherical debris of radius $R_{S}$, for different values of the geometry parameters $\chi=R_{S} / R_{B}$ and $\lambda_{r}=r_{c} / R_{B}$, where $z_{c}, r_{c}$ is the position of the center of the sphere, and $R_{B}$ is the radius of the $95 \%$-flux streamtube of the beam at $z_{c}$. Except for the small influence of streamline curvature, these results are universal.

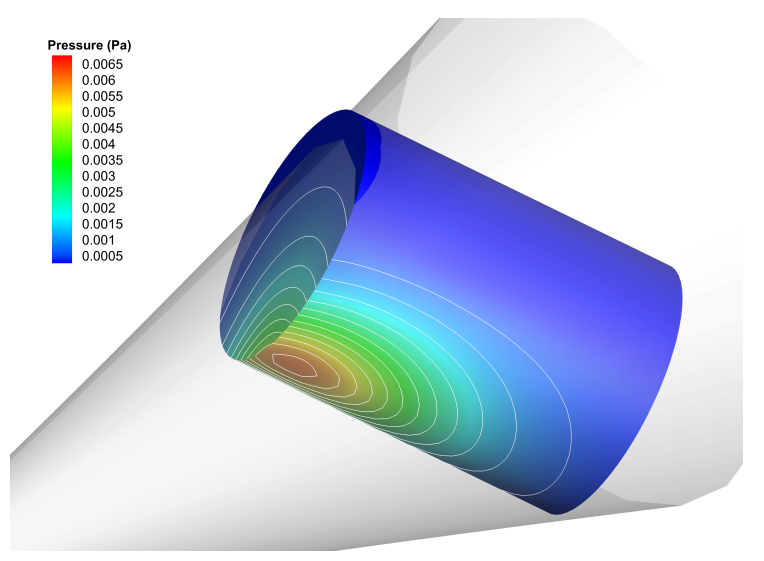

Figure 8. Momentum delivered by the ion beam upon a Kosmos upper stage (2.4 m radius, $6.5 \mathrm{~m}$ high) located at a distance of $15 \mathrm{~m}$ from the IBS, calculated with the IBIS software. An ion beam with $M=20$ and an initial divergence angle of $\arctan (1 / \tilde{d})=10 \mathrm{deg}$ was used for the simulation.

\section{A. Relative charging of the IBS-debris system}

There are a number of processes that act simultaneously on an orbiting body ${ }^{25,26}$ which tend to modify its electric charge, including (1) the presence of a background plasma, which gives immersed objects a negative potential relative to plasma potential; (2) electron photoemission of sunlit surfaces; (3) secondary electron emission from impacting particles ${ }^{27}$ (mainly energetic electrons); and (4) singular events related to energetic electron beams such as magnetic substorms. ${ }^{28}$

All these processes compete with each other, leading to an equilibrium debris potential $\phi_{D}$. For a typical satellite at altitudes below two Earth radii, the effect of ambient plasma dominates $\left(T_{e} \sim 1 \mathrm{eV} ; n \sim\right.$ $10^{10} \mathrm{~m}^{-3}$ ) and the satellite potential is slightly negative (floating potential), leaving aside singular events which may temporarily perturb the nominal situation.

In the case under analysis, $\phi_{D}$ is set mainly by the plasma beam, which is much denser than background plasma $\left(n>10^{12} \mathrm{~m}^{-3}\right)$. The object interaction with this plasma is similar to the interaction with the ambient plasma: the body tends to acquire a negative floating potential, in the order of $\sim 5 T_{e} / e$ for a xenon plasma. The potential fall is located around the body in a thin non-neutral plasma sheath, of a thickness in the order of the Debye Length of the plasma $\left(\lambda_{D}=\sqrt{\varepsilon_{0} T_{e} /\left(e^{2} n\right)}<1 \mathrm{~cm}\right.$ for $n=10^{12}$ and $\left.T_{e}=1 \mathrm{eV}\right)$. The velocity of the $\sim 1 \mathrm{keV}$ ions is still much smaller than the characteristic velocity of $\sim 1 \mathrm{eV}$ electrons $\left(u_{e} / u_{i} \simeq 15\right)$, therefore essentially not affecting this charging process nor the equilibrium potential. Since the conducting plasma beam electrically links the two bodies, they readily reach an equilibrium situation, where a relative potential between both objects is established. This relative potential results from the fall 
at the plasma sheaths of each object, and the small plasma potential fall that occurs along the beam (of the order of electron temperature $\sim T_{e}$ ), as the plasma transforms its remaining thermal energy into ion kinetic energy.

The relative situation is very stable, since the influence of the ambient plasma and photoemission effects can be neglected due to the large density of the thruster plume. Any perturbation or initial charge will be damped out by a fast transient current in the plasma bridge that connects both bodies, promoted by the large mobility of electrons. Moreover, due to the small distances between the objects, any extraneous effect that would tend to modify the potential of one body would similarly affect the other one, thus not modifying much the relative charging.

This qualitative analysis of the charging physics suggests that relative charging effects have negligible influence on the IBS concept. The quantitative assessment of the relative charging, the equilibrium voltage, and the study of the transient processes is currently under progress.

\section{B. Alteration of the plasma flow due to an immersed body}

The presence of a body in the plasma plume modifies it with the appearance of the aforementioned sheaths, which form in the beam-illuminated side of the debris. Ions entering this sheath are accelerated towards the body, yielding their momentum to it when they reach its surface. Although in this electrostatic acceleration no extra momentum is gained by the plasma-debris system, the presence of the sheath around the debris has the beneficial side effect of attracting additional lateral ions that would otherwise not intercept the body, thus slightly increasing the exerted force. This effect can be accounted for by considering the body-plus-sheath volume as an "effective" volume for the force computations. The influence, however, of a sheath thinner than $1 \mathrm{~cm}$ on a body with a characteristic length larger than $1 \mathrm{~m}$, is negligible.

The immersed object influences more dramatically the development of the plume downstream from it, by generating a wake in the flow. Inside the wake, plasma density is much lower than outside from it (typically, 2 orders of magnitude less ${ }^{25,29}$ ). Hence, the contribution to the momentum transfer of the debris surface not directly illuminated by the beam can be neglected in first approximation.

\section{Backscattering and target material sputtering}

When the IBS is correctly pointed towards the target, the majority of high-velocity ions reach the debris surface, penetrating the material substrate while losing their energy through random-walk collisions. After traveling a distance of typically a few nanometers below the surface, the normal velocity of these ions reaches zero, completing the momentum transfer process. Most of these particles are then rejected from the material lattice, and abandon the surface with velocities in the order of the wall thermal speed, which is orders of magnitude lower than the velocity of the ion beam, since

$$
T_{w} \simeq 300 K \simeq 0.02 \mathrm{eV} \ll 1 \mathrm{keV} .
$$

Hence, the contribution to momentum transfer of the backscattered particles is negligible.

On the other hand, the process of energy exchange through collisions can cause a number of the material atoms to leave the debris surface, giving rise to the so-called sputtering phenomenon, whose influence on the momentum transfer needs also to be assessed. An atom can leave the lattice when it acquires an energy greater than the surface binding energy. The sputtering of a surface under the action of a particle beam is described by the sputtering yield, defined as the mean number of sputtered atoms per incident ion. Other magnitudes of interest are the mean energy of the sputtered particles, and the angular distribution of these. All these parameters depend on the type of incoming ions and the material of the debris, the ion beam energy, and the incidence angle.

Figure 9 shows the value of the average sputtering yield and energy computed for xenon ions impacting against aluminum as a function of the ion energy. The plots have been computed numerically with TRIM. ${ }^{30}$ Because the average energy of the sputtered particles is more than two order of magnitudes smaller than the energy of the impinging ions, the effect of the momentum of the sputtered material on the momentum transfer to the debris can be neglected like backscattered xenon.

\section{Backflow contamination}

The intrusion of a debris body in the plasma plume may complicate the satellite environment, as a result of the interaction of the incoming beam with the outgoing ions and neutrals from the debris (backscattered 



Figure 9. Average sputtering yield (left) and energy per sputtered particle (right) for xenon ions impacting against an aluminum substrate at different energies.

and sputtered material).

This environment can affect negatively the performance of the IBS subsystems through various interactions, namely ${ }^{18}$ (1) the appearance of parasitic currents to biased surfaces, (2) degradation of solar array panels, thermal control and other surfaces, or (3) attenuation and refraction of electromagnetic wave transmission and reception due to the enhanced plasma density.

The use of plasma thrusters involves backflow contamination risks by itself. The two most relevant processes are (1) charge-exchange collisions (CEX) between ions and not-ionized propellant, which produce slow ions that might return onto the spacecraft if the radial electric field pushes them towards e.g. solar panels, and (2) deposition of thruster material particles sputtered from thruster walls that can become charged. ${ }^{15,17,18,31}$

These hazards are normally accounted for in satellite design, mainly by skewing beam axes away from solar panels and other sensitive equipment at a certain angle, so that satellite life is not substantially affected. ${ }^{15}$ However, for the IBS concept, it is important also to assess the formation of CEX ions due to collisions with the neutral xenon atoms returning from the debris, and the effect of incoming sputtered material.

Xenon ions do not pose a serious contamination hazard for exposed satellite surfaces such as solar panels, although they might give rise to parasitic current flows between biased surfaces. A low-pressure cloud of neutral xenon gas and particles is expected to occur naturally around the debris surface irradiated by the beam. As they expand into vacuum, these neutrals may give rise to CEX ions close to the debris surface.

Typical CEX cross-sections can be approximated with the following fitting formula: ${ }^{32,33}$

$$
\sigma_{C E X}\left(\AA^{2}\right)=A-B \log (E),
$$

where $A, B$ are parameters to be found in table 1 , and $E$ is the ion energy in $\mathrm{eV}$.

\begin{tabular}{|c|c|c|}
\hline & $\mathrm{A}\left(\AA^{2}\right)$ & $\mathrm{B}\left(\AA^{2}\right)$ \\
\hline \hline$X e^{+}+X e$ & $87.3 \pm 0.9$ & $13.6 \pm 0.6$ \\
\hline$X e^{2+}+X e$ & $45.7 \pm 1.9$ & $8.9 \pm 1.2$ \\
\hline
\end{tabular}

Table 1. Fitting parameters for CEX collisions. ${ }^{32}$

Detailed quantification of the importance of this effect requires knowledge of the neutral density $n_{n}$. However, it can be anticipated that for an IBS acting on a debris sufficiently far from the IBS, ion densities near the surface are low enough to neglect the formation of CEX ions. A rough estimate of the importance of the formation of slow ions downstream $(z \simeq 10 \mathrm{~m})$ for $M_{0}=20, \delta_{0}=0.2$ and $n_{0}=10^{16} \mathrm{~m}^{-3}$ may be performed using formula 19 , which for $E_{i} \simeq 1000 \mathrm{eV}$ yields $\sigma_{C E X} \simeq 46.5 \cdot 10^{-20} \mathrm{~m}^{-2}$.

Assuming that at the surface of the body the flux of neutrals equals that of incoming ions, $n_{n} \simeq 200 n_{i} \simeq$ $2.42 \cdot 10^{15} \mathrm{~m}^{-3}$, and the CEX ion source rate is:

$$
S_{C E X}=n_{n} n_{i} v_{r e l} \sigma_{C E X} \simeq 5.2 \cdot 10^{14} \mathrm{~m}^{-3} \mathrm{~s}^{-1},
$$

which is a low particle source rate and far away located compared to other CEX sources, ${ }^{15}$ so that it can be neglected for most practical purposes. 
Moreover, due to the large distance, most CEX ions occurring far downstream in the plume are expected to be pushed away radially with an angle large enough from the centerline as to not come back upon the satellite (additionally, plasma sheath electric potential might be beneficial as it pulls those ions and hinders their backstreaming). These assumptions and the exact behavior of neutrals and CEX ions need to be studied and clarified in more detail in the future.

Similar fluxes of sputtered material from the debris can be expected, as the sputtering yield is in the order of unity (see figure 9). Assuming a spherical expansion from the surface of a sphere of $\sim 1 \mathrm{~m}$ radius (material leaves the debris in every direction as it expands into vacuum), sputtered material flux would be $\sim 100$ times lower when it reaches the IBS (at $\sim 10 \mathrm{~m}$ distance from the debris). This means a flux of $\sim 10^{15}$ $\mathrm{m}^{-2} s^{-1}$. Assessment of the effects of this flux on the IBS lifetime requires improving this coarse estimate and detailed study of particle-surface interactions at the IBS.

\section{Conclusions}

In this paper, two key aspects of a novel active space debris removal concept, the Ion Beam Shepherd, have been analyzed, namely the requisites and characteristics of the intermediate ion beam for momentum transfer to a far body, and the different phenomena associated with the plasma-debris interaction.

In the first regard, we have identified the importance of a small beam divergence, high specific impulse, and high thrust efficiency as key performances of the desired plasma source. Off-the-shelf ion thrusters are suggested as the best option currently available, although other thruster types can be also employed for this application. Future thruster design with high $I_{s p}$, high ion Mach number, and low initial beam divergence angle as main requirements would largely benefit the performances of the Ion Beam Shepherd concept. The ion beam characterization has been carried out with the help of a quasi-selfsimilar plasma plume expansion model, which presents a good accuracy while allowing semi-analytical treatment. Quasi-selfsimilar models and the effects of the environmental plasma and magnetic field in the development of the plasma plume will be investigated in detail in a future publication. ${ }^{21}$

With respect to the plasma-debris interaction, a procedure to calculate the momentum transferred to the target body by the plasma has been described, and implemented numerically into the IBIS software. The momentum transfer efficiency, or the fraction of momentum flux intercepted by the target, has been characterized in the simple case of a spherical debris, with respect to the form-factor of the sphere to the beam radius, and the radial position of the sphere relative to the plume centerline. More debris geometries are currently being studied.

Electrically-charging phenomena have been studied qualitatively, to the conclusion that the relative charge between shepherd and debris will be very stable due to the presence of the linking high-density plasma beam and the small separation between the two bodies. Backscattering and debris sputtering have been assessed, and a preliminary analysis of backflow contamination has been carried out.

This is an ongoing research effort, and a considerable amount of work remains to be done. Specifically, finer modeling of the plasma beam and detailed assessment of these interactions and other advanced topics such as the formation of a low-density, non-neutral plasma wake in the shadow of the debris and the modification of the plasma beam in the region neighboring the immersed object need to be performed. We are currently working on the implementation of more advanced numerical models to deal with these points, which would also allow consideration of the particularities of different thruster types. The relative orbital dynamics and control of the shepherd-debris system will be addressed in a companion article. ${ }^{7}$

\section{Acknowledgments}

The work for this paper was supported by the "ARIADNA Call for Ideas on Active Debris Removal", established by the Advanced Concepts Team of the European Space Agency, under contract No. 4000101447 / 10 / NL / CBi.

\section{References}

\footnotetext{
${ }^{1}$ Liou, J. and Johnson, N., "A sensitivity study of the effectiveness of active debris removal in LEO," Acta Astronautica, Vol. 64, No. 2-3, 2009, pp. 236-243.

${ }^{2}$ Kessler, D. and Cour-Palais, B., "Collision frequency of artificial satellites: The creation of a debris belt," Journal of
} 
Geophysical Research, Vol. 83, No. A6, 1978, pp. 2637-2646.

${ }^{3}$ Bombardelli, C. and Peláez, J., "Ion Beam Shepherd for Contactless Space Debris Removal," Journal of Guidance, Control, and Dynamics, Vol. 34, No. 3, May 2011, pp. 916-920.

${ }^{4}$ Bombardelli, C., Urrutxua, H., Merino, M., Ahedo, E., Pelaez, J., and Olympio, J., "Dynamics of Ion-beam-propelled space debris," 22nd International Symposium on Space Flight Dynamics, 2011.

${ }^{5}$ Kitamura, S., "Large Space Debris reorbiter using ion beam irradiation," 61st International Astronautical Congress, International Astronautical Federation, Paris, France, 2010.

${ }^{6}$ Ruault, J. M., Desjean, M. C., Bonnal, C., and Bultel, P., "From identification of problematics to in Flight demonstration preparation," First European Workshop on Active Debris Removal, 2010.

${ }^{7}$ Bombardelli, C., Urrutxua, H., Pelaez, J., Merino, M., Ahedo, E., and Summerer, L., "Space Debris Removal with an Ion Beam Shepherd Satellite: Dynamics and Control," to be presented in the 62nd International Astronautical Congress, International Astronautical Federation, Paris, France, 2011.

${ }^{8}$ Martinez-Sanchez, M. and Pollard, J. E., "Spacecraft Electric Propulsion - An Overview," Journal of Propulsion and Power, Vol. 14, No. 5, Sept. 1998, pp. 688-699.

${ }^{9}$ Ahedo, E. and Merino, M., "Two-dimensional supersonic plasma acceleration in a magnetic nozzle," Physics of Plasmas, Vol. 17, No. 7, 2010, pp. 073501.

${ }^{10}$ Heimerdinger, D. and Martinez-Sanchez, M., "Fluid mechanics in a magnetoplasmadynamic thruster," 20th International Electric Propulsion Conference, Electric Rocket Propulsion Society, Fairview Park, OH, 1988.

${ }^{11}$ Nakles, M. R., Brieda, L., Garrett, D. R., Hargus, W. A. J., and Spicer, R. L., "Experimental and Numerical Examination of the BHT-200 Hall Thruster Plume," 43rd AIAA/ASME/SAE/ASEE Joint Propulsion Conference ES Exhibit, AIAA, Washington DC, 2007.

${ }^{12}$ Narasimha, R., "Collisionless expansion of gases into vacuum," Journal of Fluid Mechanics, Vol. 12, No. 02, Feb. 1962, pp. 294-308.

${ }^{13}$ Roberts, L. and South Jr, J., "Comments on exhaust flow field and surface impingement," AIAA Journal, Vol. 2, No. 5, 1964, pp. 971-973.

${ }^{14}$ Korsun, A. G. and Tverdokhlebova, E. M., "The characteristics of the EP exhaust plume in space," 33rd AIAA/ASME/SAE/ASEE Joint Propulsion Conference \& Exhibit, AIAA, Washington DC, 1997.

${ }^{15}$ Goebel, D. M. and Katz, I., Fundamentals of Electric Propulsion: Ion and Hall Thrusters, JPL, 2008.

${ }^{16}$ Ashkenazy, J. and Fruchtman, A., "Plasma Plume Far Field Analysis," 27th International Electric Propulsion Conference, Electric Rocket Propulsion Society, Fairview Park, OH, 2001.

${ }^{17}$ Boyd, I. D., "Review of Hall Thruster Plume Modeling," Journal of Spacecraft and Rockets, Vol. 38, No. 3, 2001, pp. 381-387.

${ }^{18}$ Roy, R. I. S., Gastonis, N. a., and Hastings, D. E., "Ion-thruster plume modeling for backflow contamination," Journal of Spacecraft and Rockets, Vol. 33, No. 4, July 1996, pp. 525-534.

${ }^{19}$ Mikellides, I. G., Jongeward, G. A., Gardner, B. M., Katz, I., Mandell, M. J., and Davis, V. A., "A Hall-Effect Thruster Plume and Spacecraft Interactions Modeling Package," 27th International Electric Propulsion Conference, Electric Rocket Propulsion Society, Fairview Park, OH, 2001.

${ }^{20}$ Passaro, A., Vicini, A., and Biagioni, L., "3-D computation of plasma thruster plumes," 40th AIAA/ASME/SAE/ASEE Joint Propulsion Conference \&S Exhibit, AIAA, Washington DC, 2004.

${ }^{21}$ Merino, M., Ahedo, E., Bombardelli, C., Urrutxua, H., and Peláez, J., "Hypersonic Plasma Plume Expansion in Vacuum and Preliminary Assessment of Magnetic Effects," to be presented in the 32nd International Electric Propulsion Conference, No. IEPC-2011-086, Electric Rocket Propulsion Society, Fairview Park, OH, 2011.

${ }^{22}$ Haas, J. M. and Gallimore, A. D., "An Investigation of Internal Ion Number Density and Electron Temperature Profiles in a Laboratory-Model Hall Thruster," 36th AIAA/ASME/SAE/ASEE Joint Propulsion Conference Es Exhibit, AIAA, Washington DC, 2000.

${ }^{23}$ Takegahara, H., Kasai, Y., Gotoh, Y., Miyazaki, K., Hayakawa, Y., Kitamura, S., Nagano, H., and Nakamaru, K., "Beam characteristics evaluation of ETS-VI Xenon ion thruster," 23rd International Electric Propulsion Conference, Vol. 7, Electric Rocket Propulsion Society, Fairview Park, OH, 1993.

${ }^{24}$ Walker, R., Bramanti, C., Sutherland, O., Boswell, R., Charles, C., Fearn, D., Del Amo, J., Frigot, P., and Orlandi, M., "Initial experiments on a dual-stage 4-grid ion thruster for very high specific impulse and power," 42nd AIAA/ASME/SAE/ASEE Joint Propulsion Conference \& Exhibit, AIAA, Washington DC, 2006.

${ }^{25}$ Garrett, H. and a.C. Whittlesey, "Spacecraft charging, an update," IEEE Transactions on Plasma Science, Vol. 28, No. 6, 2000, pp. 2017-2028.

${ }^{26}$ Rosen, A., "Spacecraft Charging by Magnetospheric plasmas," IEEE Transactions on Nuclear Science, Vol. 23, No. 6, 1976, pp. 1762-1768.

${ }^{27}$ Frederickson, A., Cotts, D., Wall, J., and Bouquet, F., Spacecraft dielectric material properties and spacecraft charging, AIAA, 1986.

${ }^{28}$ Akasofu, S., "The development of the auroral substorm," Planetary and Space Science, Vol. 12, No. 4, 1964, pp. $273-282$.

${ }^{29}$ Hastings, D. and Garrett, H., Spacecraft-environment interactions, Cambridge University Press, 2004.

${ }^{30}$ Ziegler, J. F., Biersack, J. P., and Ziegler, M. D., SRIM - The Stopping and Range of Ions in Matter, Lulu, 2007.

${ }^{31}$ Wang, J., Brinza, D., and Young, M., "Three-Dimensional Particle Simulations of Ion Propulsion Plasma Environment for Deep Space 1," Journal of Spacecraft and Rockets, Vol. 38, No. 3, 2001, pp. 433-440.

${ }^{32}$ Miller, J. S., Pullins, S. H., Levandier, D. J., Chiu, Y.-h., and Dressler, R. a., "Xenon charge exchange cross sections for electrostatic thruster models," Journal of Applied Physics, Vol. 91, No. 3, 2002, pp. 984.

${ }^{33}$ Rapp, D. and Francis, W., "Charge exchange between gaseous ions and atoms," The Journal of Chemical Physics, Vol. 37, 1962, pp. 2631. 